\title{
Gastric Adenocarcinoma in a 16-Year-Old Female
}

\author{
Kannan Periasamy, MD, DNB, ${ }^{1}$ Nikhila Radhakrishna, MD, DNB, ${ }^{1}$ Ashutosh Mukherji, MD, ${ }^{1}$ \\ Rajesh Nachiappa Ganesh, MD, DNB ${ }^{2}$
}

${ }^{1}$ Department of Radiotherapy, Regional Cancer Centre, Jawaharlal Institute of Postgraduate Medical Education and Research, Puducherry, India ${ }^{2}$ Department of Pathology, Jawaharlal Institute of Postgraduate Medical Education and Research, Puducherry, India

Background: Primary gastric adenocarcinoma commonly occurs in the elderly in the sixth to seventh decade of life but is exceedingly rare in children and adolescents, accounting for only $0.05 \%$ of pediatric gastrointestinal malignancies. Because of the nonspecific clinical presentation, gastric adenocarcinomas in children and adolescents are often metastatic when they are discovered.

Case Report: We describe a case of gastric adenocarcinoma in a 16-year-old female who was diagnosed early and successfully treated with surgery and chemoradiation by volumetric modulated arc technique.

Conclusion: The possibility of gastric adenocarcinoma should be considered in children and adolescents with persistent nonspecific symptoms of vomiting and upper abdominal pain so that the diagnosis can be made at early stages and radical treatment can be offered.

Keywords: Adolescent, chemotherapy, gastric cancer, pediatrics, radiotherapy-conformal

Address correspondence to Ashutosh Mukherji, MD, Department of Radiotherapy, Regional Cancer Centre, Jawaharlal Institute of Postgraduate Medical Education and Research, Puducherry, 605006, India. Tel: +91-948-914-6747. Email: drashutoshm@gmail.com

\section{INTRODUCTION}

Primary gastric adenocarcinoma is a disease of the elderly that usually affects patients in the sixth to seventh decade of life. ${ }^{1}$ It is uncommon in individuals $<40$ years of age ${ }^{2}$ and is exceedingly rare in children and adolescents. ${ }^{3}$ Because of the rarity and nonspecific clinical features of gastric adenocarcinoma, the diagnosis is often delayed in children and young people and is generally made at the time of emergency explorative laparotomy for acute abdomen, resulting in an adverse prognosis. ${ }^{2}$ We report the case of a 16-year-old patient with gastric adenocarcinoma who was diagnosed early and successfully treated with surgery and chemoradiation.

\section{CASE REPORT}

A 16-year-old female presented with complaints of recurrent vomiting and upper abdominal pain for 3 months. The vomiting occurred approximately 30 minutes to 1 hour after food intake, generally after solids. She had poor appetite and undocumented weight loss. The family history was negative for gastric carcinoma. The patient's abdominal examination was normal with no organomegaly. Upper gastrointestinal endoscopy showed an ulcer with a clean base in the prepyloric region with gross edema of the pyloric region. Biopsy of the ulcer revealed gastric adenocarcinoma. Contrast-enhanced computed tomography of the thorax and abdomen 3 weeks after presentation revealed homogeneous circumferential mural thickening involving the antropyloric region of the stomach with a maximum thickness of
$2.2 \mathrm{~cm}$ (Figure 1). No significant perigastric lymphadenopathy was noted; fat planes surrounding the organs appeared to be maintained. The patient had no evidence of disseminated intraabdominal disease or lymphadenopathy and no evidence of metastasis to the liver, lung, or any other organ.

Distal gastrectomy with D1 lymph node dissection and gastrojejunostomy were performed within 7 weeks of the patient's initial presentation. Postoperative histopathologic examination revealed diffuse-type adenocarcinoma infiltrating the muscularis propria and reaching the serosa (Figure 2). Proximal, distal, and circumferential margins were all free of tumor. The pathologist identified 9 lymph nodes in the resected surgical specimen, all of which were free of tumor. Postoperatively, the patient received 1 cycle of capecitabine $\left(1,000 \mathrm{mg} / \mathrm{m}^{2}\right.$ twice daily), followed by chemoradiotherapy with capecitabine $\left(825 \mathrm{mg} / \mathrm{m}^{2}\right.$ twice daily), followed by 2 cycles of capecitabine $\left(1,000 \mathrm{mg} / \mathrm{m}^{2}\right.$ twice daily). The radiation dose was $45 \mathrm{~Gy}$ in 25 fractions at $1.8 \mathrm{~Gy} /$ fraction by volumetric modulated arc technique (Figure 3). The patient was scheduled for follow-up every 3-4 months for the first 2 years, every 6 months for the next 3 years, and annual follow-up thereafter. At 10-month follow-up, the patient was asymptomatic with no evidence of locoregional disease.

\section{DISCUSSION}

In children and adolescents, gastrointestinal tract malignancies are uncommon and constitute $1.2 \%$ of their malignancies, with lymphomas and sarcomas being the most 


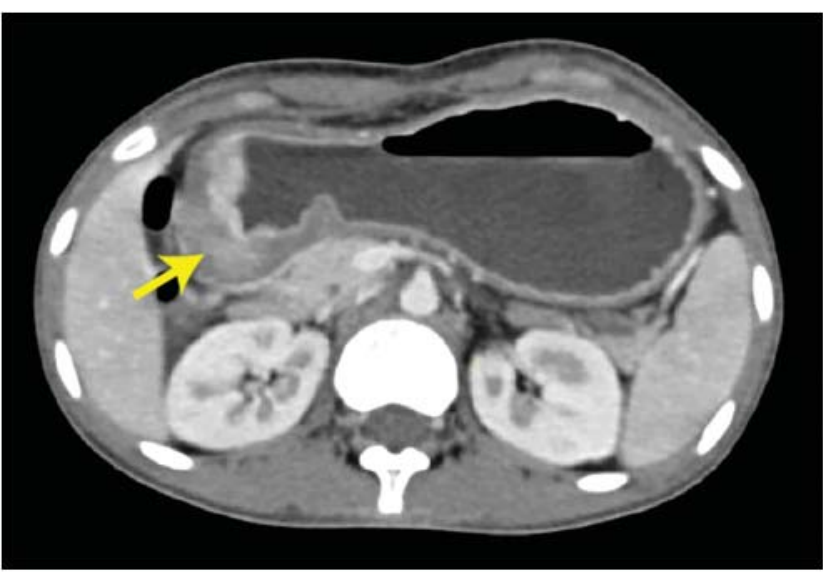

Figure 1. Contrast-enhanced computed tomography of the abdomen shows antropyloric thickening of the stomach (arrow).

common histologies., ${ }^{3,4}$ Primary gastric adenocarcinomas are exceedingly rare and constitute $0.05 \%$ of pediatric gastrointestinal malignancies. ${ }^{4}$ Only approximately 30 cases of pediatric and adolescent gastric carcinoma have been reported in the literature. ${ }^{1}$ Hence, little is known about its etiology, clinical features, treatment, and outcome. ${ }^{5}$

Helicobacter pylori infection and lifestyle factors are most commonly implicated in the etiology of adult gastric carcinomas. However, the influence of these factors in children and adolescents is less known; an inherited mutation in the
E-cadherin gene has been identified in pediatric and adolescent gastric carcinomas. ${ }^{6,7}$ Our patient was not tested for this mutation because of financial reasons. The most common clinical features of pediatric and adolescent gastric cancer include abdominal pain and vomiting that mimic other diseases of the abdomen. The diagnosis of primary gastric adenocarcinoma is often delayed in children and adolescents because of its rarity and nonspecific clinical presentation. As a result, the cases are often metastatic at the time of diagnosis, precluding curative treatment. ${ }^{1,8}$ In our case, the patient had persistent vomiting and abdominal pain for 3 months that was unresponsive to conservative management, highlighting the need to perform upper gastrointestinal endoscopy in children and adolescents with persistent symptoms of upper abdominal pain and vomiting.

The paucity of evidence and guidelines for management of pediatric and adolescent primary gastric cancer necessitates the extrapolation of management principles from adults. Patients with localized disease are managed using a multimodal approach, whereas those with metastatic disease are treated with palliative chemotherapy. ${ }^{7,8}$ Gastric cancer in children and adolescents has a poor prognosis compared to adults because the cases are often metastatic at presentation. ${ }^{9}$ The median survival after diagnosis is approximately 5 months. ${ }^{2}$

\section{CONCLUSION}

Although primary gastric adenocarcinomas are rare in children and adolescents, the possibility should be considered in patients with persistent symptoms of upper abdominal
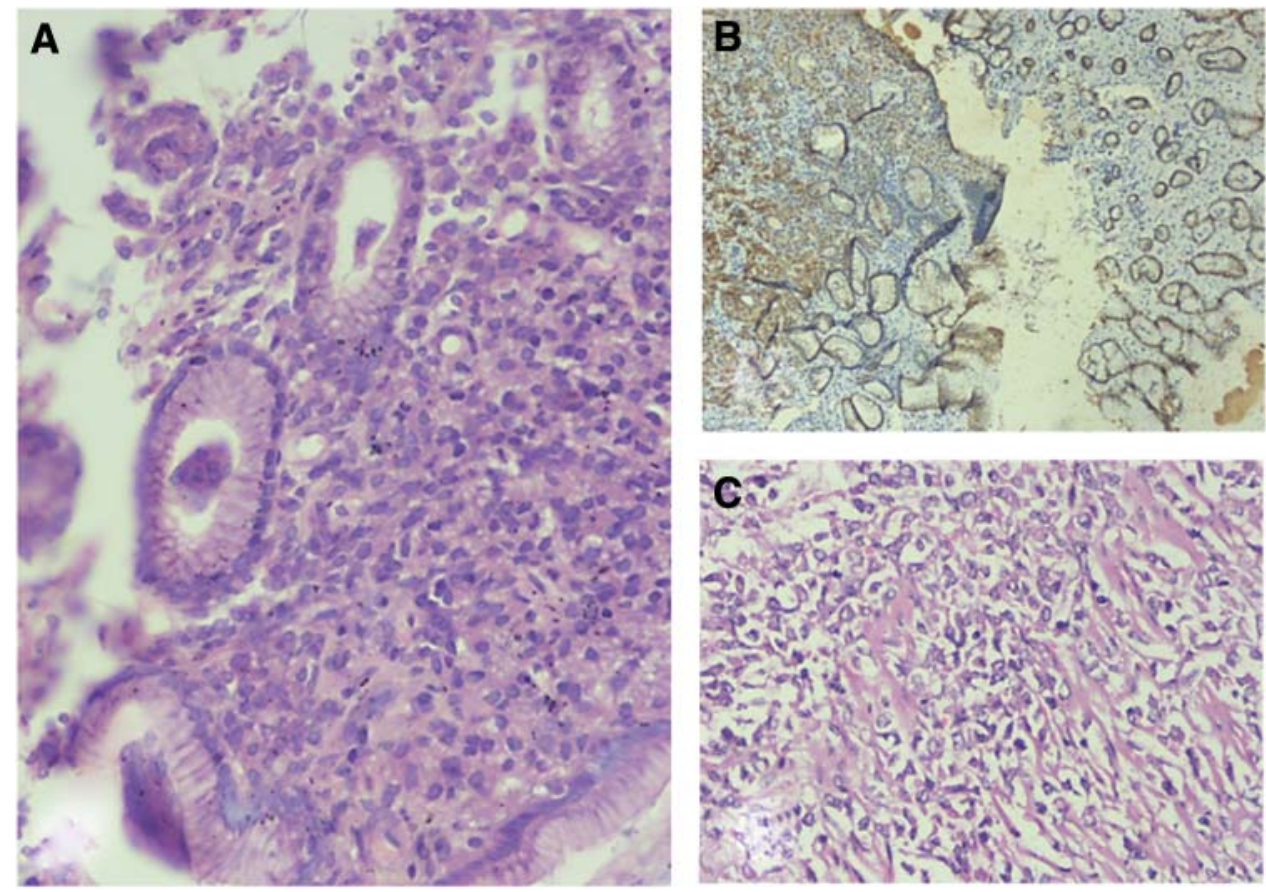

Figure 2. Histopathology. A. Gastric mucosa is infiltrated by sheets of tumor cells with signet ring cell morphology exhibiting abundant intracytoplasmic mucin (hematoxylin and eosin stain, $\times 200$ ). B. Tumor cells infiltrate the submucosa of the stomach, highlighted by pan cytokeratin immunohistochemistry (diaminobenzidine stain, DAKO monoclonal antihuman antibody, $\times 100)$. C. Tumor cells exhibit abundant intracytoplasmic mucin infiltrating the muscular layer (hematoxylin and eosin stain, $\times 200$ ). 

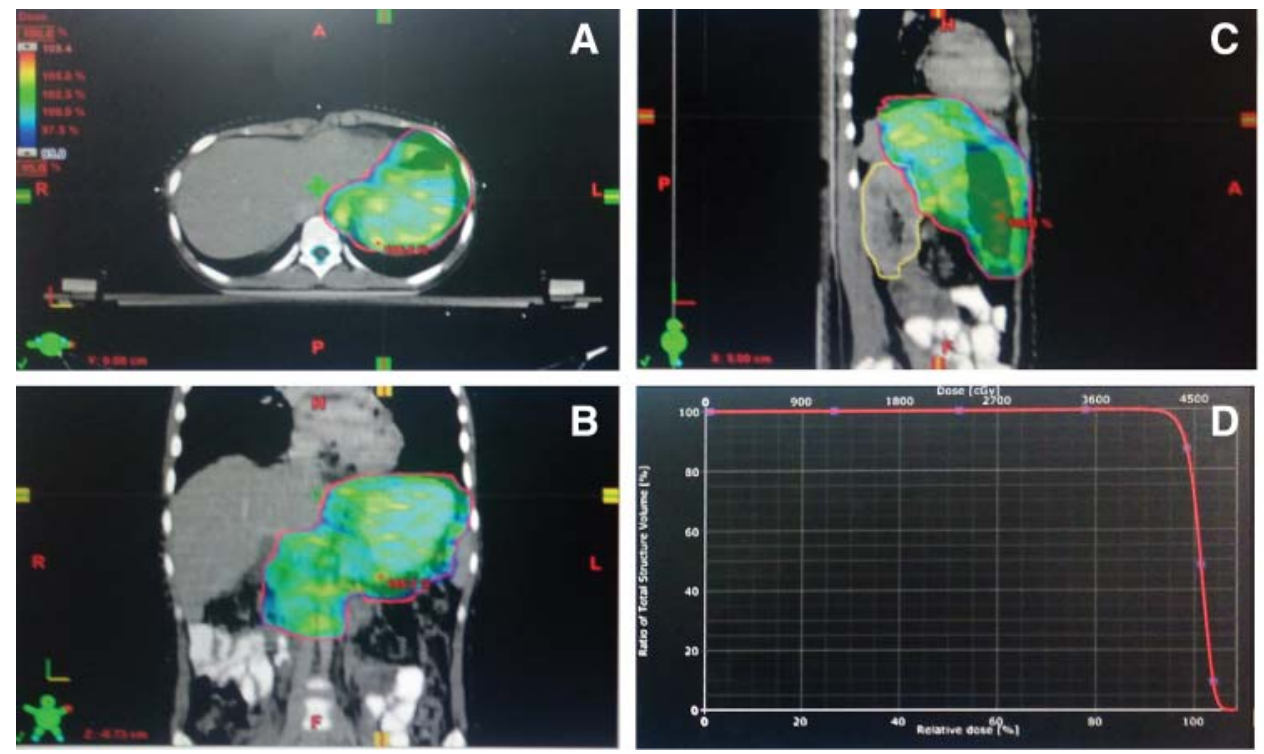

Figure 3. Conformal radiotherapy planning by volumetric modulated arc technique showing dose distribution in (A) axial, (B) coronal, and (C) sagittal planes, as well as (D) the dose-volume histogram.

pain and vomiting so that the diagnosis can be made at an early stage, and radical treatment can be offered.

\section{ACKNOWLEDGMENTS}

The authors have no financial or proprietary interest in the subject matter of this article.

\section{REFERENCES}

1. Al-Hussaini A, Alghamdi S, Alsaaran R, Al-Kasim F, Habib Z, Ourfali N. Gastric adenocarcinoma presenting with gastric outlet obstruction in a child. Case Rep Gastrointest Med. 2014;2014:527471. doi: 10.1155/2014/527471.

2. Brecht IB, Graf N, Schweinitz D, Frühwald MC, Bielack SS, Schneider DT. Networking for children and adolescents with very rare tumors: foundation of the GPOH Pediatric Rare Tumor Group. Klin Padiatr. 2009 May-Jun;221(3):181-185. doi: 10.1055/ s-0029-1220705.

3. Theuer CP, de Virgilio C, Keese G, et al. Gastric adenocarcinoma in patients 40 years of age or younger. Am J Surg. $1996 \mathrm{Nov;172}$ (5):473-476; discussion 476-477.
4. Goldthorn JF, Canizaro PC. Gastrointestinal malignancies in infancy, childhood, and adolescence. Surg Clin North Am. 1986 Aug;66(4):845-861.

5. Subbiah V, Varadhachary G, Herzog CE, Huh WW. Gastric adenocarcinoma in children and adolescents. Pediatr Blood Cancer. 2011 Sep;57(3):524-527. doi: 10.1002/pbc.23051.

6. de Martel C, Franceschi S. Infections and cancer: established associations and new hypotheses. Crit Rev Oncol Hematol. 2009 Jun;70(3):183-194. doi: 10.1016/j.critrevonc.2008.07.021.

7. Gayther SA, Gorringe KL, Ramus SJ, et al. Identification of germline E-cadherin mutations in gastric cancer families of European origin. Cancer Res. 1998 Sep 15;58(18):4086-4089.

8. Mlkvý P. Multimodal therapy of gastric cancer. Dig Dis. 2010;28 (4-5):615-618. doi: 10.1159/000320063.

9. Harting MT, Blakely ML, Herzog CE, Lally KP, Ajani JA, Andrassy RJ. Treatment issues in pediatric gastric adenocarcinoma. $J$ Pediatr Surg. 2004 Aug;39(8):e8-10.

This article meets the Accreditation Council for Graduate Medical Education and the American Board of Medical Specialties Maintenance of Certification competencies for Patient Care and Medical Knowledge. 